\title{
PROPRIÉTÉS SPECTROSCOPIQUES ET EFFET LASER D'UN VERRE TELLURITE ET D'UN VERRE PHOSPHATE FORTEMENT DOPÉS EN NÉODYME
}

\author{
J. C. MICHEL, D. MORIN et F. AUZEL. \\ Centre National d'Etudes des Télécommunications, 196, rue de Paris, 92220 Bagneux, France
}

(Reçu le 30 mai 1978, révisé le 25 août 1978, accepté le 18 septembre 1978)

\begin{abstract}
Résumé. - On présente quelques propriétés spectroscopiques, de verres dopés, importantes pour la conception de mini-lasers telles que : forces d'oscillateur, section. efficace laser, durée de vie radiative et rendement quantique en fonction de la concentration. Deux verres, un tellurite de lithium et un phosphate de cadmium potassium assez fortement dopés en néodyme. (concentration respective 0,91 et $0,73 \times 10^{21}$ at. $\mathrm{Nd}^{3+} \mathrm{cm}^{-3}$ ) sont considérés. On vérifie que ces verres ont une grande section efficace laser, soit $\sigma_{1} \sim 0,45 \times 10^{-20} \mathrm{~cm}^{2}$, moins de deux fois plus faible que celle d'un monocristal d'ultraphosphate de néodyme, et que les processus de désexcitation non radiatifs du niveau ${ }^{4} \mathrm{~F}_{3 / 2}$ en fonction de la concentration sont relativement faibles ce qui en fait des matériaux comparables aux cristaux stæchiométriques recherchés actuellement pour la mise au point des systèmes minilasers. Nous confirmons les conclusions que l'on peut tirer des résultats spectroscopiques en montrant que ces verres peuvent manifester l'effet laser en continu dans des structures optiques compactes et lorsqu'ils sont pompés longitudinalement à la longueur d'onde de $5145 \AA$. Nous trouvons en particulier que le verre tellurite a un seuil laser de $3,12 \mathrm{mV}$ absorbés par \% de pertes.
\end{abstract}

\begin{abstract}
Some spectroscopic properties of $\mathrm{Nd}^{3+}$ doped glasses in relation with mini-laser action are presented; namely; oscillator strenghts, laser cross section, radiative lifetime, quantum yield with respect concentration. Two types of glasses, a lithium tellurite one and a cadmium potassium phosphate one, respectively doped with $0.91 \times 10^{21}$ and $0.73 \times 10^{21} \mathrm{Nd}^{3+} \mathrm{cm}^{-3}$, are considered. The laser cross section is verified to be about $0.45 \times 10^{-20} \mathrm{~cm}^{2}$, that is less than half the value for Neodymium pentaphosphate (NdPP). Also self-quenching is found to be small and comparable to the one of NdPP. CW laser action is obtained for a longitudinally Argon pumped (5 $145 \AA$ ) compact structure, with a laser threshold as low as $3.12 \mathrm{~mW}$ absorbed power per $\%$ loss in the tellurite glass case.
\end{abstract}

1. Introduction. - Actuellement beaucoup d'efforts sont accomplis dans plusieurs laboratoires du monde pour mettre en évidence des matériaux lasers susceptibles d'être utilisés dans des structures compactes en vue de réaliser des mini-lasers pour télécommunications optiques.

De tels matériaux doivent posséder une caractéristique principale : à savoir un très faible taux d'autoextinction de la fluorescence pour une forte concentration $\rho$ en ions actifs $\left(\rho \geqslant 10^{21}\right.$ at. $\left.\mathrm{cm}^{-3}\right)$. Jusqu'à ce jour, exception faite des travaux de Y. K. Voronko et al. [1a-b], les matériaux qui ont été proposés [2-7] sont des monocristaux dont l'ultraphosphate de néodyme $\left(\mathrm{NdP}_{5} \mathrm{O}_{14}\right)$, mis en évidence par Danielmeyer et autres [2], est le prototype.

Nous montrons dans cet article que des verres ont cette propriété de posséder un rendement quantique relativement élevé pour une concentration importante en $\mathrm{Nd}^{3+}$. Tel est le cas pour certains tellurites, comme ceux étudiés par Singh et al. [8], qui possèdent une forte section efficace d'émission, et surtout certains phosphates, dont l'étude faite au C.N.E.T. par Deutschbein et al. [9] fait apparaître des raies d'émission fines significatives également de sections efficaces importantes [10-11].

Nous présentons ici quelques propriétés spëctroscopiques; forces d'oscillateur, section efficace laser, rendement quantique, durée de vie radiative, de deux verres : un tellurite de lithium $\left(\mathrm{V}_{1}\right)$ et un phosphate de cadmium potassium $\left(\mathrm{V}_{2}\right)$.

Nous donnons en outre les résultats des expériences laser que nous avons effectuées avec ces deux matériaux.

2. Préparations des verres. - Les deux matrices vitreuses dopées au $\mathrm{Nd}^{3+}$ que nous avons plus spécialement étudiées répondent aux formules suivantes :

Verre tellurite $\left(\mathrm{V}_{1}\right) \quad\left\{\begin{array}{l}\mathrm{TeO}_{2}: 95,53 \% \\ \mathrm{Li}_{2} \mathrm{O}: 4,47 \%\end{array}\right.$ (en poids) 
Verre phosphate $\left(\mathrm{V}_{2}\right)\left\{\begin{array}{l}\mathrm{P}_{2} \mathrm{O}_{5}: 59,60 \% \\ \mathrm{Na}_{2} \mathrm{O}: 5,66 \% \\ \mathrm{~K}_{2} \mathrm{O}: 22,44 \% \\ \mathrm{CdO}: 12,30 \% .\end{array}\right.$ (en poids)

En ajoutant, à la masse de chacun des mélanges cidessus, respectivement $5 \%$ et $8 \%$ de cette masse en poids de $\mathrm{Nd}_{2} \mathrm{O}_{3}$, nous obtenons une concentration de $0,91 \times 10^{21}$ et $0,73 \times 10^{21}$ at. $\mathrm{Nd}^{3+} \cdot \mathrm{cm}^{-3}$ pour $V_{1}$ et $V_{2}$ (densité respective 5,34 et $2,78 \mathrm{~g} . \mathrm{cm}^{-3}$ ).

Les produits utilisés pour les préparations, outre l'oxyde de néodyme (Rhône-Poulenc), pureté 99,999, sont respectivement pour $\mathrm{V}_{2}$, l'anhydride phosphorique $\mathrm{P}_{2} \mathrm{O}_{5}$, les carbonates $\mathrm{Na}_{2} \mathrm{CO}_{3}, \mathrm{~K}_{2} \mathrm{CO}_{3}, \mathrm{CdCO}_{3}$ (Prolabo) qualité RP pour analyșes, et pour $\mathrm{V}_{1}$, le carbonate de lithium $\mathrm{Li}_{2} \mathrm{CO}_{3}$ (BLB) pour analyses, et l'oxyde de tellure $\mathrm{TeO}_{2}$ (Merck) pureté 99,999 (LAB).

Dans le cas des verres phosphates, les produits mélangés en poudre sont introduits petit à petit (à cause de la libération d'anhydride phosphorique) dans un creuset de platine, chauffé dans un four à moufle à $800^{\circ} \mathrm{C}$. Le creuset est ensuite transféré dans un four à rhodium, chauffé jusqu'à $1300^{\circ} \mathrm{C}$ et maintenu à cette température pendant deux heures pour le débullage et l'homogénéisation du verre.

Le coulage a lieu alors dans un moule en graphite préchauffé, et un recuit de trois heures environ à $300^{\circ} \mathrm{C}$ termine la préparation en supprimant les tensions internes dues à la trempe.

Dans le cas des verres tellurites, les produits mélangés sont placés dans un creuset de platine également, mais en une seule fois, chauffés dans un four à moufle à $500^{\circ} \mathrm{C}$ pendant $30 \mathrm{~min}$., puis dans un four à rhodium une heure à $900^{\circ} \mathrm{C}$.

Le coulage a toujours lieu dans un moule en graphite préchauffé, mais le recuit est fait à $200^{\circ} \mathrm{C}$ pendant $20 \mathrm{~h}$, le verre tellurite étant beaucoup plus fragile que le verre phosphate. Les températures de recuit ont été déterminées par analyse thermique différentielle.

3. Absorption. - La figure 1 donne les spectres de l'absorption entre 0,9 et $0,4 \mu \mathrm{m}$ de $V_{1}$ et $V_{2}$. Ces spectres sont obtenus à $300 \mathrm{~K}$ à l'aide d'un spectrophotomètre CARY 17R. Les échantillons utilisés ont respectivement pour épaisseur $6 \times 10^{-2} \mathrm{~cm}$ et $5,25 \times 10^{-2} \mathrm{~cm}$.

De ces enregistrements nous déterminons les forces d'oscillateurs correspondant aux transitions d'absorption à partir du niveau fondamental, nous en déduisons les paramètres $T_{\dot{\lambda}}$ qui apparaissent dans la théorie de Judd [12] (les résultats sont présentés dans le tableau I) et nous remontons ensuite aux forces d'oscillateur entre niveaux excités [13] ce qui nous permet de calculer la section efficace intégrée [10]

$$
\sigma_{1} \cdot \Delta v_{\text {eff }}=\int \sigma(v) \mathrm{d} v
$$

puis $\sigma_{1}$ lorsque l'on a déterminé $\Delta v_{\text {eff }}$.

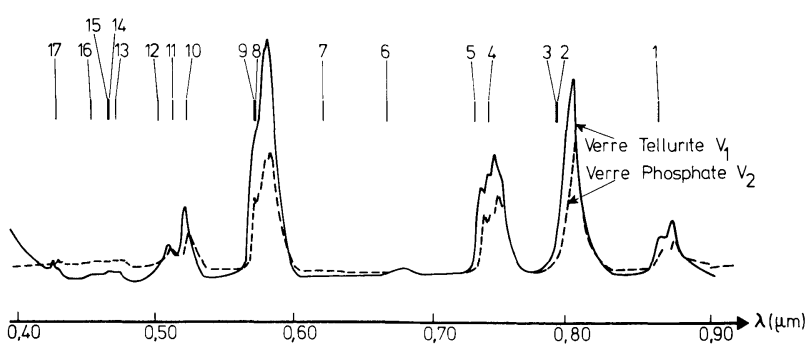

Fig. 1. - Spectre d'absorption du verre tellurite $\mathrm{V}_{1}$ et du verre phosphate $\mathrm{V}_{2}$. Epaisseur : $6 \times 10^{-2}$ et $5,25 \times 10^{-2} \mathrm{~cm}$. Les nombres se réfèrent à la position des niveaux d'énergie de l'ion libre de $\mathrm{Nd}^{3+}$ comme cela a été calculé par Wybourne (J. Chem. Phys. 639 (1960) 32 1960). 1, ${ }^{4} \mathrm{~F}_{3 / 2} ; 2,{ }^{4} \mathrm{~F}_{5 / 2} ; 3,{ }^{2} \mathrm{H}_{9 / 2} ; 4,{ }^{4} \mathrm{~S}_{3 / 2}$; $5,{ }^{4} \mathrm{~F}_{7 / 2} ; 6,{ }^{4} \mathrm{~F}_{9 / 2} ; 7,{ }^{2} \mathrm{H}_{11 / 2} ; 8,{ }^{2} \mathrm{G}_{7 / 2} ; 9,{ }^{4} \mathrm{G}_{5 / 2} ; 10,{ }^{2} \mathrm{~K}_{13 / 2} ; 11$, ${ }^{4} \mathrm{G}_{7 / 2} ; 12,{ }^{4} \mathrm{G}_{9 / 2} ; 13,{ }^{2} \mathrm{~K}_{15 / 2} ; 14,{ }^{2} \mathrm{D}_{3 / 2} ; 15,{ }^{2} \mathrm{G}_{9 / 2} ; 16,{ }^{4} \mathrm{G}_{11 / 2}$;

$$
17,{ }^{2} \mathrm{P}_{1 / 2} \text {. }
$$

[Absorption spectra of lithium tellurite glass, $\mathrm{V}_{1}$, and cadmium phosphate glass $\mathrm{V}_{2}$. Thickness : $6 \times 10^{-2}$ and $5.25 \times 10^{-2} \mathrm{~cm}$. Numbers refer to positions of energy levels of $\mathrm{Nd}^{3+}$ free ion as calculated by Wybourne (J. Chem. Phys. 639 (1960) 32). 1, ${ }^{4} \mathrm{~F}_{3 / 2}$; $2,{ }^{4} \mathrm{~F}_{5 / 2} ; 3,{ }^{2} \mathrm{H}_{9 / 2} ; 4,{ }^{4} \mathrm{~S}_{3 / 2} ; 5,{ }^{4} \mathrm{~F}_{7 / 2} ; 6,{ }^{4} \mathrm{~F}_{9 / 2} ; 7,{ }^{2} \mathrm{H}_{11 / 2} ; 8,{ }^{2} \mathrm{G}_{7 / 2}$; $9,{ }^{4} \mathrm{G}_{5 / 2} ; 10,{ }^{2} \mathrm{~K}_{13 / 2} ; 11,{ }^{4} \mathrm{G}_{7 / 2} ; 12,{ }^{4} \mathrm{G}_{9 / 2} ; 13,{ }^{2} \mathrm{~K}_{15 / 2} ; 14,{ }^{2} \mathrm{D}_{3 / 2}$; $\left.15,{ }^{2} \mathrm{G}_{9 / 2} ; 16,{ }^{4} \mathrm{G}_{11 / 2} ; 17,{ }^{2} \mathrm{P}_{1 / 2}.\right]$

\section{TABLEAU I}

Forces d'oscillateur $(f)$ et paramètres de JUDD $\left(T_{\lambda}\right)$ dans les verres tellurites $\mathrm{V}_{1}$ et phosphate $\mathrm{V}_{2}$

[Oscillators strengths $(f)$ and Judd's parameters for lithium tellurite glass $\left(\mathrm{V}_{1}\right)$ and cadmium phos-

\begin{tabular}{|c|c|c|}
\hline \multirow[b]{2}{*}{${ }^{4} \mathrm{I}_{9 / 2} \rightarrow$} & \multicolumn{2}{|c|}{$f .10^{6}$} \\
\hline & Tellurite & Phosphate \\
\hline - & - & - \\
\hline${ }^{4} \mathrm{~F}_{3 / 2}$ & 1,48 & 1,7 \\
\hline${ }^{4} \mathrm{~F}_{5 / 2},{ }^{2} \mathrm{H}_{9 / 2}$ & 5,2 & 6,2 \\
\hline${ }^{4} \mathrm{~S}_{3 / 2},{ }^{4} \mathrm{~F}_{7 / 2}$ & 4,8 & 5,9 \\
\hline${ }^{4} \mathrm{~F}_{9 / 2}$ & 0,36 & 0,43 \\
\hline${ }^{2} \mathrm{G}_{7 / 2},{ }^{4} \mathrm{G}_{5 / 2}$ & 14,6 & 14,6 \\
\hline${ }^{2} \mathrm{~K}_{13 / 2},{ }^{4} \mathrm{G}_{7 / 2},{ }^{4} \mathrm{G}_{9 / 2}$ & 4,53 & 5,14 \\
\hline${ }^{2} \mathrm{~K}_{15 / 2},{ }^{2} \mathrm{D}_{3 / 2},{ }^{2} \mathrm{G}_{9 / 2},{ }^{4} \mathrm{G}_{11 / 2}$ & 1,01 & 3,6 \\
\hline${ }^{2} \mathrm{P}_{1 / 2}$ & 0,36 & \\
\hline $\mathrm{T}_{2} \times 10^{21} \mathrm{~s}^{-1}$ & 37,45 & 24,16 \\
\hline $\mathrm{T}_{4} \times 10^{21} \mathrm{~s}^{-1}$ & 29,26 & 24,03 \\
\hline $\mathrm{T}_{6} \times 10^{21} \mathrm{~s}^{-1}$ & 34,15 & 19,18 \\
\hline
\end{tabular}
phate glass $\left(\mathrm{V}_{2}\right)$.]

4. Fluorescence. - Les spectres d'émission, figure 2 , sont relatifs à la transition ${ }^{4} \mathrm{~F}_{3 / 2} \rightarrow{ }^{4} \mathrm{I}_{11 / 2}$. Ils ont été obtenus à $300 \mathrm{~K}$, au moyen d'une installation classique comprenant un monochromateur Jarrell-Ash $(0,5 \mathrm{~m})$ associé à une détection synchrone P.A.R. L'excitation lumineuse de l'échantillon, modulée à $85 \mathrm{~Hz}$ par un disque tournant, est réalisée par la radiation à $5145 \AA$ d'un laser à argon ionisé (Spectra, Physics 164). La fluorescence émise par l'échantillon est recueillie à la sortie du monochro- 


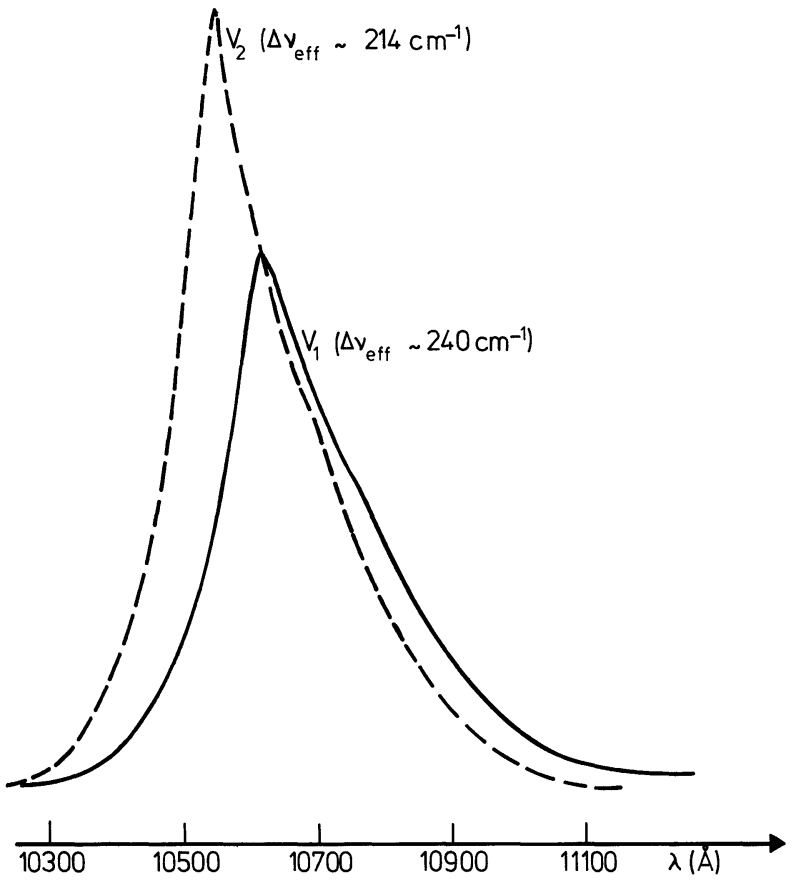

Fig. 2. - Spectre d'émission de $V_{1}$ et $V_{2}$. Transition ${ }^{4} F_{3 / 2} \rightarrow{ }^{4} I_{11 / 2}$. Résolution : $10 \AA$.

[Fluorescence spectra of $V_{1}$ and $V_{2}$. Transition ${ }^{4} F_{3 / 2} \rightarrow{ }^{4} I_{11 / 2}$. Resolution : $10 \AA$.

mateur par une cellule au sulfure de plomb non refroidie.

Pour chacun des spectres enregistrés nous déterminons la largeur de raie effective [9] [14] :

$$
\Delta v_{\text {eff }}=\frac{\int(v) \mathrm{d} v}{I_{\max }}
$$

soit : $\Delta v_{\text {eff }} \sim 240$ et $214 \mathrm{~cm}^{-1}$ respectivement pour $V_{1}$ et $V_{2}$. Ces mesures permettent de calculer la section efficace laser de chacun de ces deux verres. On a en effet [10], [16].

$$
\begin{aligned}
\sigma_{1}=\frac{1}{\Delta v_{\text {eff }}} \frac{\pi e^{2}}{m c^{2}} & \left(\frac{2 J_{0}+1}{2 J_{1}+1}\right) v \times \\
& \times \sum_{\lambda} T_{\lambda}\left|\left\langle{ }^{4} \mathrm{~F}_{3 / 2}\left\|U^{(\lambda)}\right\|{ }^{4} \mathrm{I}_{11 / 2}\right\rangle\right|^{2}
\end{aligned}
$$

où $J_{0}=9 / 2, J_{1}=3 / 2$.

$m$ et $e$ sont la masse et la charge de l'électron $v$ la fréquence de la transition laser. Les éléments de matrice $\left|\left\langle\left\|U^{(\lambda)}\right\|\right\rangle\right|^{2}$ ont été calculés par ailleurs [10], [16], $c$ est la vitesse de la lumière dans le vide.

Nous obtenons

$$
\begin{array}{lll}
\sigma_{1}=4,7 \times 10^{-20} \mathrm{~cm}^{2} & \text { pour } & \mathrm{V}_{1} \text { (tellurite) } \\
\sigma_{1}=4,4 \times 10^{-20} \mathrm{~cm}^{2} & \text { pour } & \mathrm{V}_{2} \text { (phosphate) }
\end{array}
$$

La valeur de la section efficace laser de ces deux verres est importante et supporte la comparaison avec celle de l'ultraphosphate de néodyme NdLaUP considéré jusqu'ici comme le prototype des matériaux pour mini-laser. En effet, en utilisant la méthode de calcul exposée ci-dessus pour déterminer $\sigma_{1}$, méthode qui se justifie lorsque l'on ne connaît pas la structure fine des niveaux d'énergie ${ }^{4} \mathrm{~F}_{3 / 2}$ et ${ }^{4} \mathrm{I}_{11 / 2}$ [15], nous obtenons $\sigma_{1} \sim 8,25 \times 10^{-20} \mathrm{~cm}^{2}$ pour NdLaUP avec $\Delta v_{\text {eff }}=127 \mathrm{~cm}^{-1}$. Mais, pour que ces types de verres puissent être considérés comme des matériaux possibles pour des systèmes mini-laser, il faut que le phénomène d'auto extinction de l'émission issue de ${ }^{4} \mathrm{~F}_{3 / 2}$ soit aussi réduit que possible en fonction de la concentration ou, ce qui revient au même, que les processus non radiatifs qui désexcitent ${ }^{4} \mathrm{~F}_{3 / 2}$ au fur et à mesure que l'on augmente le pourcentage en ions actifs restent faibles. On a une idée de l'importance de ces phénomènes en suivant l'évolution de la durée de vie $\tau_{\mathrm{m}}$ de ${ }^{4} \mathrm{~F}_{3 / 2}$ en fonction de la concentration puisqu'en effet on a :

$$
\left[\tau_{\mathrm{m}}\right]^{-1}=W_{\mathrm{R}}+W_{\mathrm{NR}} \quad \text { où } \quad W_{\mathrm{R}}=\left[\tau_{\mathrm{R}}\right]^{-1}
$$

est la probabilité d'émission radiative $\left(\tau_{R}\right.$ étant la durée de vie radiative) indépendante de la concentration et $W_{\mathrm{NR}}$ est la probabilité de désexcitation non radiative de ${ }^{4} \mathrm{~F}_{3 / 2}$ dépendante, elle, de la concentration.

La figure 3 présente les résultats obtenus pour $\mathrm{V}_{1}$ et $\mathbf{V}_{2}$. Nous y avons joint la courbe $\tau=f(\rho)$ relative à l'ultraphosphate de néodyme. Pour effectuer les

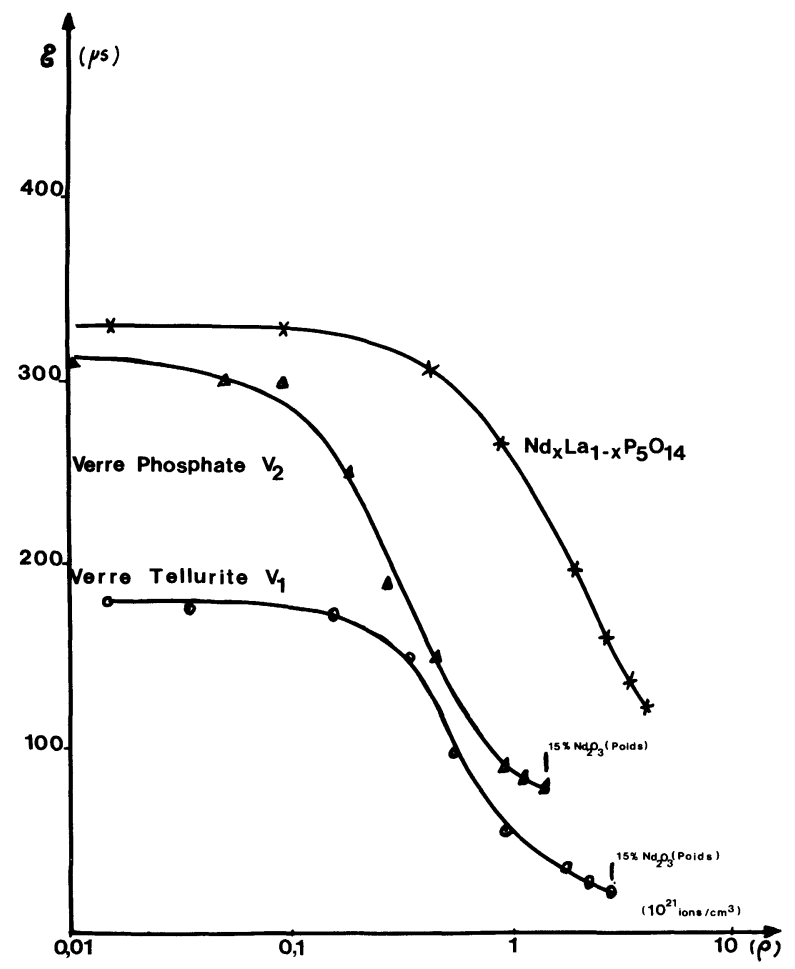

Fig. 3. - Courbe de la durée de vie de ${ }^{4} \mathrm{~F}_{3 / 2}$ en fonction de la concentration du verre tellurite $V_{1}$, du verre phosphate $V_{2}$ et de $\mathrm{Nd}_{x} \mathrm{La}_{1-x} \mathrm{P}_{5} \mathrm{O}_{14}$.

[Concentration variation of the fluorescent lifetime of the $\mathrm{Nd}^{3+}$ ${ }^{4} \mathrm{~F}_{3 / 2}$ state in tellurite glass phosphate glass and $\mathrm{Nd}_{x} \mathrm{La}_{1-x} \mathrm{P}_{5} \mathrm{O}_{14}$.] 
mesures de $\tau_{\mathrm{m}}$, les échantillons sont excités par l'intermédiaire d'une diode laser GaAlAs qui délivre des impulsions de $\simeq 10 \mathrm{~W}$ ayant 200 à $300 \mathrm{~ns}$ de largeur avec une fréquence de $\simeq 1 \mathrm{kHz}$. Ces impulsions, dont la longueur d'onde est égale à $8100 \AA$, pompent les niveaux ${ }^{2} \mathrm{H}_{9 / 2},{ }^{4} \mathrm{~F}_{5 / 2}$ de $\mathrm{Nd}^{3+}$. La fluorescence émise par l'échantillon est filtrée puis détectée par un photomultiplicateur (couche sensible $S_{1}$ ) dont le signal électrique est amplifié puis traité par un Waveform Eductor de P.A.R. associé à un oscilloscope sur l'écran duquel s'inscrit la décroissance exponentielle de la fluorescence en fonction du temps.

Nous déterminons $W_{\mathbf{R}}$ par le calcul avec :

$$
W_{\mathrm{R}}=\sum_{S^{\prime}, L^{\prime}, J^{\prime}} A\left(S, L, J, S^{\prime}, L^{\prime}, J^{\prime}\right)
$$

où

$$
A\left(S, L, J ; S^{\prime}, L^{\prime}, J^{\prime}\right)
$$

est la probabilité d'émission spontanée du niveau émetteur $S, L, J\left({ }^{4} \mathrm{~F}_{3 / 2}\right)$ vers le niveau $\left(S^{\prime}, L^{\prime}, J^{\prime}\right)$, elle-même obtenue, comme $\sigma_{1}$, à partir des éléments de matrice $\left|\left\langle\left\|U^{(\hat{\lambda})}\right\|\right\rangle\right|^{2}$.

Connaissant la valeur calculée de $\tau_{\mathbf{R}}$ il est possible de vérifier si le rendement quantique $\eta$ du niveau ${ }^{4} \mathrm{~F}_{3 / 2}$ est proche de 1 pour les basses concentrations d'ions $\left(\rho<10^{19}\right.$ at. $\left.\mathrm{cm}^{-3}\right)$ auquel cas les phénomènes non radiatifs peuvent être négligeables à ces concentrations puisque :

$$
\eta=\frac{W_{\mathrm{R}}}{W_{\mathrm{R}}+W_{\mathrm{NR}}} .
$$

Au fur et à mesure que l'on augmente la concentration dans les échantillons le rendement quantique décroît mettant en évidence la croissance des phénomènes non radiatifs. Nous avons fait en sorte d'introduire dans $V_{1}$ et $V_{2}$ la concentration nécessaire en ions actifs pour atteindre une valeur du rende- ment quantique de ${ }^{4} \mathrm{~F}_{3 / 2}$ qui soit comparable à la valeur du rendement de $\mathrm{Nd}_{0,75} \mathrm{La}_{0,25} \mathrm{P}_{5} \mathrm{O}_{14}$, le matériau cristallin type optimisé pour mini-laser. Ceci afin d'avoir pour ces deux verres un taux de désexcitation non radiatif du niveau ${ }^{4} \mathrm{~F}_{3 / 2}$ équivalent.

Le tableau II regroupe les valeurs relatives à : $\tau_{\mathbf{R}}$, $\eta$ à faible concentration : $\left(\eta_{1}\right)$, à concentration pour laquelle chaque verre a été testé en effet laser : $\left(\eta_{2}\right)$ et à concentration maximale en ions actifs que nous avons introduits dans $\mathrm{V}_{1}$ et $\mathrm{V}_{2}:\left(\eta_{3}\right)$. Nous avons également porté dans ce tableau les valeurs calculées des rapports d'embranchement d'émission pour chaque transition radiative issue de ${ }^{4} \mathrm{~F}_{3 / 2}(J)$. Ces taux s'expriment par :

$$
\beta_{J J^{\prime}}=\frac{A\left(S, L, J ; S^{\prime}, L^{\prime}, J^{\prime}\right)}{\sum_{S^{\prime}, L^{\prime}, J^{\prime}} A\left(S, L, J ; S^{\prime}, L^{\prime}, J^{\prime}\right)} .
$$

L'analyse du tableau II montre l'excellent comportement des deux verres au point de vue du rendement quantique de ${ }^{4} \mathrm{~F}_{3 / 2}$ à forte concentration $(15 \%$ en poids de $\mathrm{Nd}_{2} \mathrm{O}_{3}$ ). Ce pourcentage d'oxyde de néodyme est le maximum que l'on puisse introduire dans le verre phosphate $\mathrm{V}_{2}$ sans risque de dévitrification. Pour de tels pourcentages de $\mathrm{Nd}_{2} \mathrm{O}_{3}$ la concentration est de $2,7 \times 10^{21}$ ions $/ \mathrm{cm}^{3}$ pour le verre tellurite $V_{1}$ et de $1,5 \times 10^{21}$ ions $/ \mathrm{cm}^{3}$ pour le verre phosphate $V_{2}$.

On peut noter que la durée de vie radiative $\tau_{R}$ calculée pour chaque verre ne correspond pas tout à fait à la durée de vie mesurée à faible concentration notamment pour le verre phosphate. Cela peut s'expliquer par la présence d'impuretés qui désexcitent non radiativement le niveau ${ }^{4} \mathrm{~F}_{3 / 2}$. Ainsi on sait que les conditions de préparation influent sur la durée de vie de ${ }^{4} \mathrm{~F}_{3 / 2}$ par l'intermédiaire d'une plus ou moins grande concentration en radicaux $\mathrm{O}-\mathrm{H}$ résiduels [11].

Le verre tellurite possède quant à lui une probabilité de désexcitation radiative élevée ce qui en fait, toute chose égale par ailleurs, un matériau à grand gain.

\section{TABleAU II}

Durée de vie radiative $\tau_{\mathbf{R}}$, rendement quantique à faible concentration $\eta_{1}$, à concentration pour laquelle les échantillons ont été testés en effet laser $\eta_{2}$, et à forte concentration $\eta_{3}$, de l'état ${ }^{4} \mathrm{~F}_{3 / 2}$ de $\mathrm{Nd}^{3+}$ dans le verre tellurite $\mathrm{V}_{1}$ le verre phosphate $\mathrm{V}_{2}$ et $\mathrm{NdP}_{5} \mathrm{O}_{14}$. Taux de fluorescence calculés $\beta_{4_{\mathrm{F}_{3 / 2}}} \rightarrow j, j=15 / 2$, $13 / 2,11 / 2,9 / 2$.

\begin{tabular}{|c|c|c|c|c|c|c|c|c|}
\hline Matériaux & $\begin{array}{c}\tau_{\mathrm{R}} \\
(\mu \mathrm{s}) \\
-\end{array}$ & $\eta_{1}$ & $\underline{\eta_{2}}$ & $\eta_{3}$ & $\begin{aligned} \beta & \\
{ }^{4} \mathrm{~F}_{3 / 2} & \rightarrow{ }^{4} \mathrm{I}_{15 / 2} \\
& -\end{aligned}$ & $\begin{aligned} \beta & \\
{ }^{4} \mathrm{~F}_{3 / 2} & \rightarrow{ }^{4} \mathrm{I}_{13 / 2} \\
& -\end{aligned}$ & $\begin{array}{c}\beta \\
{ }^{4} \mathrm{~F}_{3 / 2} \stackrel{{ }^{4} \mathrm{I}_{11 / 2}}{-}\end{array}$ & $\begin{aligned} & \beta \\
{ }^{4} \mathrm{~F}_{3 / 2} & \rightarrow{ }^{4} \mathrm{I}_{9 / 2}\end{aligned}$ \\
\hline Verre tellurite $\mathrm{V}_{1}$ & 199 & 0,9 & $\begin{array}{c}\left(5 \% \mathrm{Nd}_{2} \mathrm{O}_{3}\right) \\
0,28\end{array}$ & $\begin{array}{c}\left(15 \% \mathrm{Nd}_{2} \mathrm{O}_{3}\right) \\
0,12\end{array}$ & $4,59 \times 10^{-3}$ & $\times 10^{-2}$ & 0,49 & 0,41 \\
\hline $\begin{array}{l}\text { Verre phosphate alca- } \\
\operatorname{lin} V_{2}\end{array}$ & 404 & 0,77 & $\begin{array}{c}\left(8 \% \mathrm{Nd}_{2} \mathrm{O}_{3}\right) \\
0,25\end{array}$ & $\begin{array}{c}\left(15 \% \mathrm{Nd}_{2} \mathrm{O}_{3}\right) \\
0,20\end{array}$ & $3,88 \times 10^{-3}$ & $7,93 \times 10^{-2}$ & 0,45 & 0,46 \\
\hline $\begin{array}{l}\mathrm{Nd}_{0,75} \mathrm{La}_{0,25} \mathrm{P}_{5} \mathrm{O}_{14} \\
\mathrm{Nd}_{x} \mathrm{La}_{1-x} \mathrm{UP}\end{array}$ & 322 & 1 & $\begin{array}{c}(x=0,75) \\
0,43\end{array}$ & $\begin{array}{c}(x=1) \\
0,37\end{array}$ & $4,51 \times 10^{-3}$ & $9,26 \times 10^{-2}$ & 0,48 & 0,41 \\
\hline
\end{tabular}

[Fluorescence lifetime $\left(\tau_{\mathbf{R}}\right)$, quantum efficiency at low concentration $\left(\eta_{1}\right)$, at concentration for test laser $\left(\eta_{2}\right)$ and at large concentration $\left(\eta_{3}\right)$ from state ${ }^{4} \mathrm{~F}_{3 / 2}$ in tellurite glass $\left(\mathrm{V}_{1}\right)$, phosphate glass $\left(\mathrm{V}_{2}\right)$ and $\mathrm{NdP}_{5} \mathrm{O}_{14}$. Calculated branching ratios $: \beta_{4^{4} \mathrm{~F}_{3 / 2}} \rightarrow j$ with $j=15 / 2,13 / 2,11 / 2,9 / 2$.] 
5. Résultats laser. - 5.1 DisPOSITIF EXPÉRIMENTAL. - L'échantillon que l'on veut tester en effet laser, en configuration de pompage longitudinal, est placé au centre d'une cavité quasi concentrique constituée par deux miroirs de $1 \mathrm{~cm}$ de rayon de courbure séparés de $2 \mathrm{~cm}$ environ. Ces miroirs transmettent $90 \%$ de la raie à $5145 \AA$ du laser à argon dont on se sert comme source de pompage. Le miroir d'entrée de la cavité à une réflectivité maximum à $1,06 \mu \mathrm{m}$ (transmission résiduelle de l'ordre de $5 \times 10^{-4}$ ).

Le miroir de sortie a une transmission de $1 \%$ à cette longueur d'onde. Le diamètre, mesuré, du faisceau de pompe focalisé au voisinage du centre de la cavité est de l'ordre de $40 \mu \mathrm{m}$. La mesure a été effectuée au moyen d'une fente d'analyse se déplaçant avec une vitesse uniforme dans un plan normal au faisceau.

5.2 RÉSultats. - La figure 4 donne la puissance infrarouge émise aux alentours de $\lambda \sim 1,06 \mu \mathrm{m}$ en fonction de la puissance absorbée à $0,5145 \mu \mathrm{m}$ par

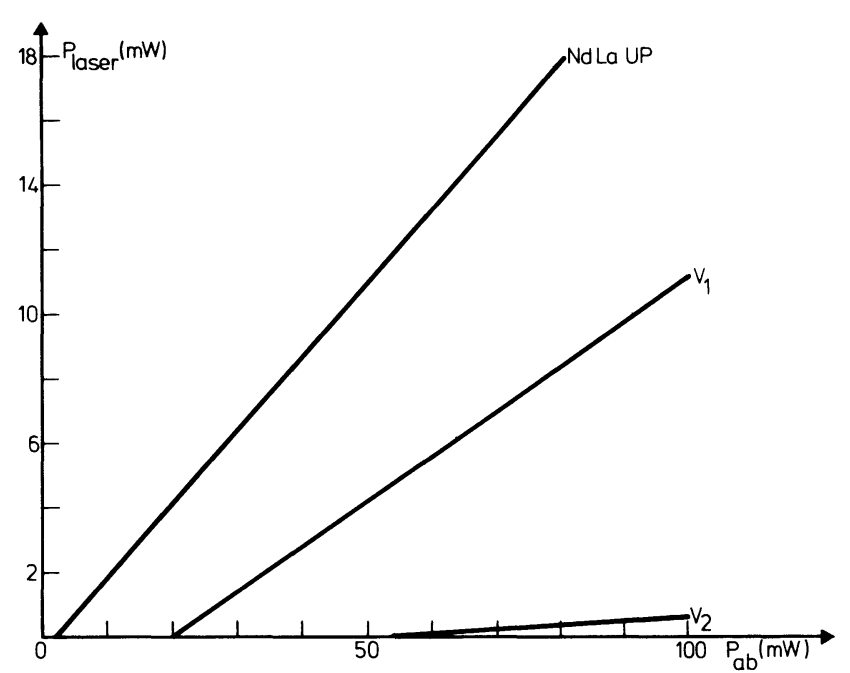

FIG. 4. - Puissance infra-rouge émise $(\lambda \sim 1,06 \mu \mathrm{m})$ en fonction de la puissance absorbée à $0,51 \mu \mathrm{m}$ par NdLaUP, $V_{1}$ et $V_{2}$.

[Infra-red $(\lambda \sim 1,06 \mu \mathrm{m})$. Power emission of NdLaUP, $\mathrm{V}_{1}$ and $\mathrm{V}_{2}$ in term from absorbed power at $0,51 \mu \mathrm{m}$.] le verre tellurite. Nous avons porté de plus sur cette figure les mesures relatives à un échantillon d'ultra-phosphate de néodyme de composition $\mathrm{Nd}_{0,75} \mathrm{La}_{0,25} \mathrm{P}_{5} \mathrm{O}_{14}$. Ceci, afin de pouvoir apprécier les pertes propres à la cavité utilisée.

En ce qui concerne le verre phosphate nous n'avons observé l'effet laser qu'avec une cavité composée de deux miroirs ayant une transmission très faible (de l'ordre de $5 \times 10^{-4}$ ) à $1,06 \mu \mathrm{m}$. Nous donnons plus loin des résultats partiels concernant ce matériau. L'épaisseur de l'échantillon testé est égale à $0,382 \mathrm{~mm}$ pour NdLaUP (orienté 001, axe $b$ contenū dans la plaquette et perpendiculaire au faisceau laser), $1,45 \overline{0} \overline{\mathrm{mm}}$ pour $\mathrm{V}_{1}$ et $1,075 \mathrm{~mm}$ pour $\mathrm{V}_{2}$.

La puissance absorbée $P_{\mathrm{ab}}$ a été déterminée en tenant compte des réflexions du faisceau de pompage qui se produisent au niveau des faces des échantillons.

$P_{\mathrm{ab}}=P_{\mathrm{i}}\left(1-t^{2} \exp (-\alpha L)\left[\frac{1}{1-r^{2} \exp (-2 \alpha L)}\right]\right)$

où $r=\left(\frac{n-1}{n+1}\right)^{2}, t \sim 1-r, n$ est l'indice de réfraction, $\alpha$ est le coefficient d'absorption à la longueur d'onde de pompage, $L$ est la longueur de l'échantillon, $P_{\mathrm{i}}$ est la puissance incidente de pompage considérée au niveau de la face d'entrée de l'échantillon.

Nous avons regroupé dans le tableau III les valeurs relatives à $n$ ainsi que celles de $\alpha$ pour $\lambda=5145 \AA$ et pour $\lambda$ situé aux alentours de $8100 \AA$ ( $\alpha$ est mesuré à $\lambda$ donné, on ne tient pas compte des largeurs de raies correspondantes).

Les puissances absorbées au seuil laser sont respectivement de $2 \mathrm{~mW}$ pour NdLaUP et $200 \mathrm{~mW}$ pour $\mathrm{V}_{1}$.

Afin de minimiser les effets thermiques produits au sein des échantillons par le faisceau de pompage fortement focalisé, celui-ci est modulé par un disque tournant (extinction du faisceau $70 \%$ par cycle).

Les énergies de pompage et d'émission I.R. sont mesurées à l'aide d'un radiomètre photomètre EG et $\mathrm{G}$ associé à un module intégrateur (550-3).

$\mathrm{Si}$ on suppose que toute l'énergie absorbée finit

\section{TABlEAU III}

Indice de réfraction et coefficient d'absorption pour $\lambda=5145 \AA$ et $\lambda=8100 \AA$ du verre tellurite $\mathrm{V}_{1}$, $d u$ verre phosphate $\mathrm{V}_{2}$ et de $\mathrm{Nd}_{0,75} \mathrm{La}_{0,25} \mathrm{P}_{5} \mathrm{O}_{14}$

[Refractive index and absorption coefficient for $\lambda=5145 \AA$ and $\lambda=8100$ from tellurite glass $\left(\mathrm{V}_{1}\right)$, phosphate glass $\left(\mathrm{V}_{2}\right)$ and $\left.\mathrm{Nd}_{0.75} \mathrm{La}_{0.25} \mathrm{P}_{5} \mathrm{O}_{14}\right]$

\begin{tabular}{|c|c|c|c|c|}
\hline & & NdLaUP & $\begin{array}{c}\mathrm{V}_{1} \\
\text { Verre tellurite } \\
-\end{array}$ & $\begin{array}{c}\mathrm{V}_{2} \\
\text { Verre phosphate } \\
-\end{array}$ \\
\hline & $n$ & 1,62 & 1,95 & 1,53 \\
\hline & $\begin{array}{l}\lambda=5145 \AA \\
\lambda \sim 8100 \AA\end{array}$ & 10,13 & 6,85 & 3,6 \\
\hline$\alpha\left(\mathrm{cm}^{-1}\right)$ & $\begin{array}{l}\text { au maximum des } \\
\text { transitions } \\
{ }^{4} \mathrm{I}_{9 / 2} \rightarrow{ }^{4} \mathrm{~F}_{5 / 2},{ }^{2} \mathrm{H}_{9 / 2}\end{array}$ & 144 & 38 & 31 \\
\hline
\end{tabular}


en émission stimulée, la pente des courbes de la figure 4 exprime le rendement quantique différentiel externe du système.

$$
\eta_{\mathrm{e}}=\eta_{\mathrm{i}} \frac{P_{\mathrm{ms}}}{P_{\mathrm{ms}}+P_{\mathrm{me}}+P_{\mathrm{m}}+P_{\mathrm{c}}}
$$

où $\eta_{\mathrm{i}}$ est le rendement quantique différentiel interne (quotient différentiel du nombre de désexcitations produites par émission stimulée au nombre total de désexcitations, au-dessus du seuil $\left.\eta_{\mathrm{i}} \sim 1\right), P_{\mathrm{ms}}$ est la perte introduite par le miroir de sortie $\left(P_{\mathrm{ms}} \sim 1 \%\right)$ $P_{\text {me }}$ est celle du miroir d'entrée $\left(P_{\text {me }} \sim 0\right) P_{\mathrm{m}}$ est la perte intrinsèque au matériau et $P_{\mathrm{c}}$ est une perte relative à la cavité optique (elle tient compte des phénomènes de diffraction, d'absorption et de diffusion des couches multidiélectriques des miroirs, etc...).

L'échantillon NdLaUP utilisé est d'excellente qualité optique, les seules pertes estimées par ailleurs [15] sont les pertes résonnantes dont l'ordre de grandeur est de $0,09 \%$ ce que nous négligeons devant $P_{\mathrm{ms}}$. Pour cet échantillon $\eta_{\mathrm{e}} \sim 0,23$ on trouve alors $P_{\mathrm{c}} \sim 3,3 \%$.

Pour $\mathrm{V}_{1}$ on a $\eta_{\mathrm{e}} \sim 0,14$ ce qui donne pour ce matériau $P_{\mathrm{m}} \sim 3,1 \%$ déduction faite de $P_{\mathrm{c}}$, supposée égale à la valeur précédente.

Pour le verre phosphate $V_{2}$ nous obtenons un seuil $P_{\mathrm{ab}}$ de $54 \mathrm{~mW}$ et une efficacité quantique $\eta_{\mathrm{e}}$ de $1,3 \times 10^{-3}$ ce qui conduit à des pertes $P_{\mathrm{m}}$ de $39 \%$ environ, compte tenu des pertes $P_{\mathrm{c}}$ (supposées être identiques aux pertes $P_{\mathrm{c}}$ propres à la cavité utilisant un miroir de sortie ayant une transmission de $1 \%$ ).

Ces résultats appellent quelques commentaires; d'abord en ce qui concerne la valeur relativement élevée que nous avons trouvée pour $\boldsymbol{P}_{\mathrm{c}}$. On interprète ces pertes comme étant dues à la diffraction du faisceau infrarouge dans la cavité laser, notamment par l'imperfection optique des miroirs. On sait en effet que plus on se rapproche de la configuration concentrique et plus le rayon du mode gaussien sur les miroirs est grand. Ainsi pour un rapport $D / R=1,95(D=$ distance séparant les miroirs) on calcule [17] que le rayon $W_{0}$ du mode fondamental au centre de la cavité est égal à $16,5 \mu \mathrm{m}$ alors que sur les miroirs il atteint la valeur $W_{\mathrm{m}}=100 \mu \mathrm{m}$. Afin de travailler avec une cavité plus stable que la cavité concentrique, nous nous sommes placés dans une configuration telle que $D / R \sim 1,6$. On calcule alors que $W_{0} \sim 28 \mu \mathrm{m}$ et $W_{\mathrm{m}} \sim 66 \mu \mathrm{m}$ ce qui assure une divergence moins importante au mode fondamental. Un examen attentif a montré que le miroir d'entrée de la cavité présente de petites rayures au voisinage de son centre. Nous attribuons les pertes $\boldsymbol{P}_{\mathrm{c}}$ principalement à la diffraction du faisceau laser par ces rayures.

Les pertes relatives au verre tellurite nous semblent minimes compte tenu du fait que l'installation de fabrication du laboratoire ne permet pas des coulées importantes de matériau, ce qui limite beaucoup la qualité optique des échantillons que l'on peut obtenir.
Par contre l'échantillon dont nous disposions était très sensible aux effets thermiques induits par les densités de pompage auxquelles il a été soumis. Nous avons observé également que ce matériau était le siège de contraintes mécaniques importantes ce qui indique que les recuits successifs que nous lui avons fait subir ne les avaient pas totalement éliminées.

En ce qui concerne le verre phosphate alcalin $\mathrm{V}_{2}$ les résultats sont donnés avec une certaine incertitude et ne reflètent pas tout à fait ce que l'on est en droit d'attendre de ce verre au vu des mesures spectroscopiques précédentes. En effet l'échantillon dont nous disposions était de mauvaise qualité optique à tel point que l'effet laser ne se manifestait que dans certaines zones du matériau. En outre, l'émission observée était très instable, due au fait que l'on n'arrivait pas à optimiser convenablement les réglages de la cavité optique. Dans ces conditions les différentes mesures que nous avons effectuées avec ce matériau ne sont données qu'à titre indicatif, afin de montrer que ce type de verre est apte à être utilisé dans des structures mini-laser.

On peut tenter un comparaison de NdLaUP et du verre tellurite en calculant le rapport de la puissance absorbée au seuil laser par \% de pertes totales (pertes de la cavité + pertes intrinsèques aux échantillons) pour chaque matériau. On a :

$$
\frac{\text { Verre tellurite }}{\text { NdLaUP }}=\frac{3,12(\mathrm{~mW})}{0,61(\mathrm{~mW})} \sim 5 \text {. }
$$

Dans cette cavité, et pour un pompage longitudinal, l'ultraphosphate de néodyme est donc 5 fois supérieur au verre tellurite.

La puissance absorbée au seuil laser, pour ce type de pompage, est inversement proportionnelle au produit $\sigma_{1} \tau_{\mathrm{m}}$ où $\sigma_{1}$ est la section efficace laser et $\tau_{m}$ la durée de vie de ${ }^{4} \mathrm{~F}_{3 / 2}$ niveau émetteur de $\mathrm{Nd}^{3+}$. Avec les valeurs numériques obtenues pour $\sigma_{1}$ et $\tau_{\mathrm{m}}$ on a :

$$
\frac{\dot{\sigma}_{1} \tau_{\mathrm{m}}(\text { tellurite })}{\sigma_{1} \tau_{\mathrm{m}}(\mathrm{NdLaUP})} \sim 0,22 .
$$

Ce qui correspond pratiquement à la valeur expérimentale déterminée ci-dessus à savoir que le seuil laser (en puissance absorbée) ramené par $\%$ de pertes totales est seulement cinq fois plus élevé dans le verre tellurite $\mathrm{V}_{2}$ que dans NdLaUP.

5.3 SPECTRES LASER. - La figure $5 a$ montre les spectres laser obtenus pour les verres 1 et 2 . Ces spectres ont été enregistrés au moyen de l'installation d'analyse de la fluorescence précédemment décrite. Comme on peut le constater l'émission laser du verre phosphate s'effectue à $1,0535 \mu \mathrm{m}$, avec une largeur à mi-hauteur de $40 \AA$, elle se trouve assez fortement décalée vers le visible par rapport à celle du verre tellurite centrée à $1,0650 \mu \mathrm{m}$ et ayant une largeur à mi-hauteur de $54 \AA ̊$ environ. 


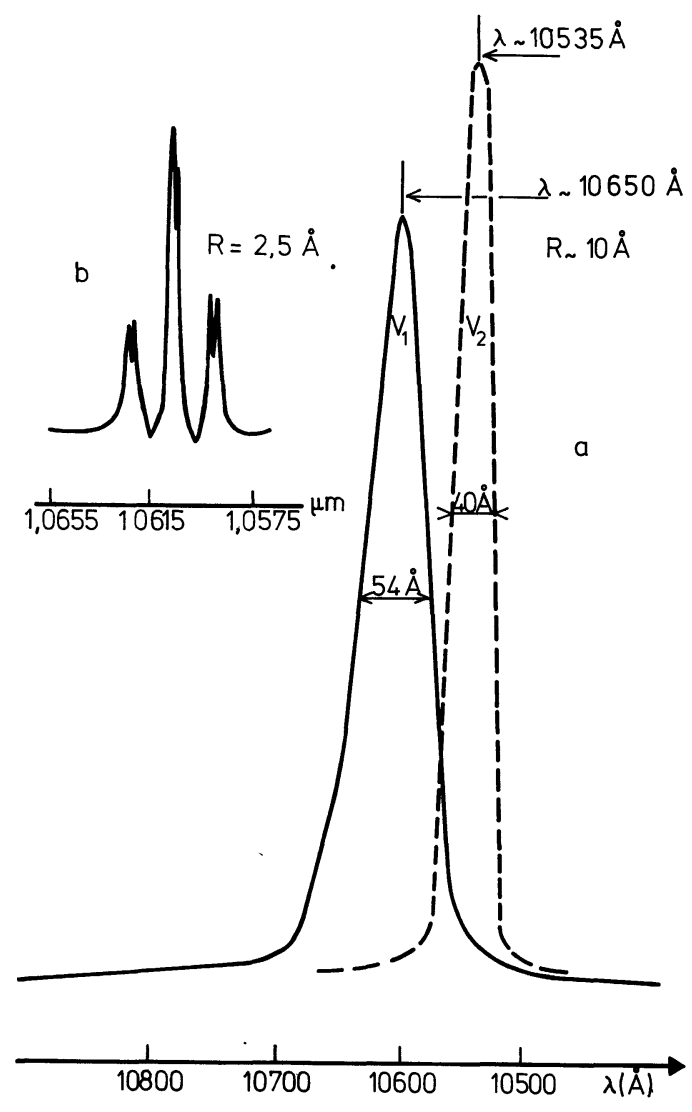

FIG. 5. - Spectre laser de $V_{1}$ et $V_{2}$. a) Résolution : $10 \AA . b$ ) Résolution : $2,5 \AA\left(\mathrm{V}_{1}\right)$.

[Laser spectra of $\mathrm{V}_{1}$ and $\mathrm{V}_{2} . a$ ) Resolution : $10 \AA . b$ ) Resolution : $\left.2,5 \AA\left(\mathrm{V}_{1}\right) \cdot\right]$

En optimisant au mieux les réglages de la cavité optique et en prenant de grandes précautions pour isoler la cavité des vibrations mécaniques nous avons pu enregistrer, pour le verre tellurite, le spectre laser présenté en figure $5 b$. On y observe que l'émission est composée de 3 raies dont la plus intense est centrée à $1,0607 \mu \mathrm{m}$. Chacune de ceś raies a une largeur à mi-hauteur de 5,5 $\AA$ environ. La résolution théorique du monochromateur était de $2,5 \AA$ lors de l'enregistrement de ces spectres.

Les largeurs spectrales importantes de l'émission laser montrées en figure $5 a$ proviennent essentiellement d'un réglage imparfait de la cavité optique qui entrâne une grande instabilité de l'émission. Dans de telles conditions celle-ci s'établit en effet, d'une manière aléatoire sur un certain nombre de modes longitudinaux et transverses, ce qui a pour conséquence d'élargir globalement en fréquence l'émission observée et de rendre impossible une analyse spectrale fine.
6. Conclusion. - Nous avons déterminé les principales propriétés spectroscopiques d'un verre tellurite et d'un verre phosphate assez fortement dopés en $\mathrm{Nd}^{3+}$. Ces propriétés, notamment la section efficace laser et la valeur du rendement quantique élevée pour des concentrations atteignant

$$
1,4 \text { à } 2,7 \times 10^{21} \text { ions } \times \mathrm{cm}^{-3},
$$

montrent que ces matrices vitreuses ont une autoextinction du niveau ${ }^{4} \mathrm{~F}_{3 / 2}$ de $\mathrm{Nd}^{3+}$, comparable à celle des monocristaux pour mini-laser comme, par exemple, l'ultraphosphate de néodyme $\mathrm{NdP}_{5} \mathrm{O}_{14}$.

De fait, l'effet laser a été obtenu avec ces deux verres dans une structure optique compacte, les échantillons étant pompés longitudinalement par la raie à $0,51 \mu \mathrm{m}$ d'un laser à Argon. Nous avons montré en particulier que le seuil laser, mesuré en puissance absorbée par \% de pertes totales, était égal à $3,12 \mathrm{~mW}$ dans le verre tellurite, ce qui est seulement cinq fois plus élevé que la puissance absorbé au seuil par un monocristal d'ultraphosphate de néodyme de composition $\mathrm{Nd}_{0,75} \mathrm{La}_{0,25} \mathrm{P}_{5} \mathrm{O}_{14}$.

Les propriétés spectroscopiques du verre phosphate alcalin sont excellentes, seule la qualité optique de l'échantillon dont nous disposions, n'a pas permis de mettre en évidence les caractéristiques laser qu'elles impliquent.

L'ensemble des résultats présentés permet d'affirmer que ces matériaux vitreux sont aptes à être utilisés dans des structures laser compactes éventuellement pour les télécommunications optiques.

Remerciements. - Nous remercions Mme C. Gautier et M. L. Dugrand pour l'aide apportée à la préparation des matériaux, Mme A. Jouanin pour le polissage des échantillons et Mlle C. Gueugnon pour la mesure des indices de réfraction.

Nota. - Alors que nous nous apprêtions à envoyer ce texte pour publication nous avons pris connaissance d'un projet de conférence et d'un article traitant tous les deux de l'effet laser dans des verres phosphates. Il s'agit :

1) De « CW oscillation of a Nd : Phosphate glass laser » conférence qui sera présentée, le 29 mai 1978 au $10^{\mathrm{e}}$ congrès d'électronique quantique qui se tiendra à Atlanta (U.S.A.) du 29 mai au $1^{\text {er }}$ juin 78, par :

Kishida, S., Washio, K. and Yoshikawa, S., Nippon Electric. Co ; Ltd., Kawasaki, Japan, and Kato Y., Osaka University, Osaka, Japan.

2) De « Spectroscopy and lasing of a high $\mathrm{Nd}$ concentration Al-phosphate glass " by LEMPICKI, A., Klein, R. M. and ChinN, S. R., I.E.E.E. J. Quant. Electron. QE 14 (1978) 283. 


\section{Bibliographie}

[1a] Voronko, Yu. K., Denker, B. I., Zlenko, A. A., KarisiK, A. Ya., KuZ'minov, Yu. S., MaKsimova, G. V., Neutruyen, V. B., Osiko, V. V., Prokhorov, A. M., Sychugov, V. A., Shipulo, G. P. and ShCherbakov, I. A., Opt. Commun. 1 (1976) 88.

[1b] Mêmes auteurs que [1a], Sov. Phys. Dokl. 21 (1976).

[2] Danielmeyer, H. G. and Heinz, Weber, P., IEEE J. Quant. Electron. QU 8 (1972) 805.

[3] Tomoaki-Yamada, Kenju-Otsuka and Jun'ichi nakano, J. Appl. Phys. 45 (1974) 5096.

[4] Chinn, S. R. and Hong, H. Y. P., Opt. Commun. 1 (1976) 87.

[5] Hong, H. Y. P. and Dwight, K., Mat. Res. Bull. 9 (1974) 775.

[6] Hong, H. Y. P. and Dwight, K., Mat. Res. Bull. 9 (1974) 1661.

[7] Michel, J. C., Mlle Morin, D. et Auzel, F., C.R. Hebd. Séan. Acad. Sci. 281B (1975) 445.

[8] Singh, S., Van Uitert, L. G. and Grodkiewicz, W. H., Opt. Commun. 17 (1976) 315.

[9] Deutschbein, O. K., Pautrat, C. C. and Svirchevsky, J. M., Revue Phys. Appl. 2 (1967) 29.
[10] Auzel, F. et Michel, J. C., C.R. Hebd. Séan. Acad. Sci. B 279 (1974) 187.

[11] Deutschbein, O. K., Pautrat, C. et Auzel, F., « Recherches sur Matériaux laser vitreux » Etude CNET no 727. P.C.M. (1964, Issy-les-Moulineaux).

[12] JudD, B. R., Phys. Rev. 127 (1962) 750.

[13] Auzel, F., Thèse (Paris, 1968) et Ann. Telecom. 24 (1969) 199.

[14] Sarkies, P. H., Sandoe, J. N. and Parke, S., Brit. J. Appl. Phys. (J. Phys. D) 4 (1971) 1642.

[15] Budin, J. P., Michel, J. C. and Auzel, F., " oscillators strengths and laser effect en $\mathrm{Na}_{2} \mathrm{Nd}_{2} \mathrm{~Pb}_{6}\left(\mathrm{PO}_{4}\right)_{6} \mathrm{Cl}_{2}(\mathrm{CLAP})$, a new-mini-laser material " à paraître dans $J$. Appl. Phys. (Décembre 1978)

[16] KRUPKE, W. F., I.E.E.E. J. Quant. Electron. QE 10 (1974) 450

[17] KogelniK, H., Modes in Optical Resonators "Lasers " édité par Albert K. Levine, Marcel Dekker (Inc., New York) vol. 1, 1966. 